Article

\title{
Analysis of Controlled Rabi Flopping in a Double Rephasing Photon Echo Scheme for Quantum Memories
}

\author{
Rahmat Ullah ${ }^{1,2}$ (D) and Byoung S. Ham ${ }^{1, *(D)}$ \\ 1 Center for Photon Information Processing, and School of Electrical Engineering and Computer Science, \\ Gwangju Institute of Science and Technology, Gwangju 61005, Korea; rahmatktk@comsats.edu.pk \\ 2 Quantum Optics Lab., Department of Physics, COMSATS University, Islamabad 44000, Pakistan \\ * Correspondence: bham@gist.ac.kr
}

Received: 19 August 2020; Accepted: 7 September 2020; Published: 9 September 2020

\begin{abstract}
A double rephasing scheme of a photon echo is analyzed for inversion-free photon echo-based quantum memories using controlled Rabi flopping, where the Rabi flopping is used for phase control of collective atom coherence. Unlike the rephasing-caused $\pi$-phase shift in a single rephasing scheme, the control Rabi flopping between the excited state and an auxiliary third state induces coherence inversion. Thus, the absorptive photon echo in a double rephasing scheme can be manipulated to be emissive. Here, we present a quantum coherence control of atom phases in a double rephasing photon echo scheme for emissive photon echoes for quantum memory applications.
\end{abstract}

Keywords: quantum optics; coherent transients; quantum memory; photon echoes

\section{Introduction}

Modified photon echoes have been intensively studied for quantum memory applications over the last decade since the first protocol of controlled reversible inhomogeneous broadening, where an efficient photon echo can be achieved by a counter-propagating control pulse set in a three-level Doppler [1] and non-Doppler medium [2]. Due to the inherent population inversion in photon echoes [3], resulting in quantum noises and violation of the no cloning theorem [4], a conventional photon echo itself cannot be directly applied to quantum memories. Compared with single-atom-based quantum memory protocols, e.g., utilizing nuclear spins recently demonstrated in Si-based semiconductors [5], the photon echoes in rare-earth doped solids have benefits of multimode, ultrafast, and ultrahigh absorption [6]. To overcome the inherent population inversion in photon echoes [3], atomic frequency comb (AFC) echoes [7,8], gradient echoes [9,10], and controlled double rephasing (CDR) echoes [11-13] are presented for quantum memory applications. Because ultralong quantum memory is an essential condition for long-distance quantum communications using quantum repeaters [14,15], storage time extension has also been a critical issue [16-19]. As experimentally demonstrated by using dynamic decoupling (DD) [16] and optical locking via controlled coherence conversion (CCC) [20], the optical storage time can be extended up to spin population decay time.

The CCC theory was proposed to convert the absorptive echo into an emissive one in a double rephasing (DR) photon echo scheme [11]. The DR photon echo scheme inherently gives the benefit of no population inversion. Because a $\pi$-rephasing pulse induces reversible coherence evolutions in a time domain with a $\pi$-phase shift, the DR echo is obviously absorptive like the data pulse due to the $2 \pi$-phase shift (no change) in the collective coherence. Regardless of silent echoes in the DR scheme [21-23], the collective coherence of the final echo is the sum of individual coherence evolutions, resulting in absorptive coherence [11,12]. Moreover, there is no way to solve this absorptive echo 
problem in a two-level system, at least not yet. It should be noted that the photon echo in an ensemble system must be distinguished from a single atomic system. Although population inversion or the sign of coherence has nothing to do with the single qubit system, it is critical to the ensemble system because of the macroscopic coherent transients of a nonlinear effect. The $\pi-\pi$ control pulse-induced negative sign of ensemble coherence can never be radiated out of the medium regardless of population inversion $[11,17,23]$.

DR photon echoes, however, have been observed, which is seemingly violating the CDR echo theory [20-22]. The reason of seemingly contradiction in [20-22] is due to the imperfect rephrasing pulse area caused by a Gaussian rephrasing pulse. Recently, such Gaussian pulse-caused echo generation was discussed to be as high as $26 \%$ in retrieval efficiency [24]. The CCC in CDR echoes was already discussed in a single rephasing photon echo scheme theoretically $[25,26]$, as well as experimentally [27]. Here, in the present paper, we analytically investigate the collective atom phase shift in the DR scheme and confirm the CDR echo theory with proof of coherence inversion. Compared with full numerical analysis in previous discussions $[11,12,17,18,25]$, we present full analytic solutions in this article.

\section{Theory}

Figure 1 is a schematic diagram of the present CDR echoes, where the control pulse set of $C_{1}$ and $C_{2}$ is for the atom phase control in the DR scheme. The data (D), first rephasing $\left(R_{1}\right)$, and second rephasing $\left(R_{2}\right)$ pulses satisfy a DR photon echo scheme, where they are resonant between states $|1\rangle$ and $|2\rangle$ as shown in Figure 1a. The pulse sequence of CDR is shown in Figure 1b, where the control pulse set $C_{1}$ and $C_{2}$ is resonant between states $|2\rangle$ and $|3\rangle$. The time delay $\tau$ between $C_{1}$ and $C_{2}$ is used for storage time extension, which is limited by the spin dephasing [25-27]. The spin dephasing can be minimized with the zero first-order Zeeman method [28]. In an optical locking scheme applied to three-pulse photon echoes [18], the storage time extends up to spin population decay time [20]. To satisfy general conditions of $C D R$, each pulse area of $R_{1}, R_{2}, C_{1}$, and $C_{2}$ is set to be $\pi$. The pulse area of $\mathrm{D}$ is set to small at $0.1 \pi$. The pulse area is defined by $\varphi_{i}=\int \Omega_{i} \mathrm{dt}$, and $\Omega_{i}\left(i=\mathrm{D}, \mathrm{R}_{1}, \mathrm{R}_{2}, \mathrm{C}_{1}\right.$, and $\left.\mathrm{C}_{2}\right)$ is the Rabi frequency of the pulse.

(a)

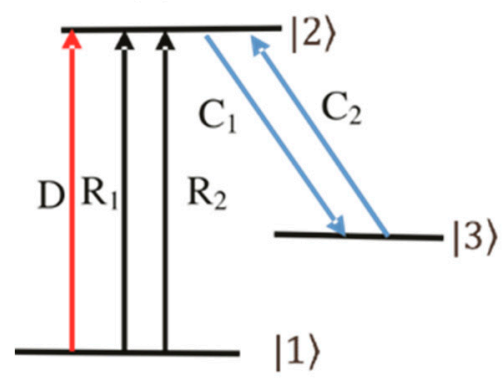

(b)

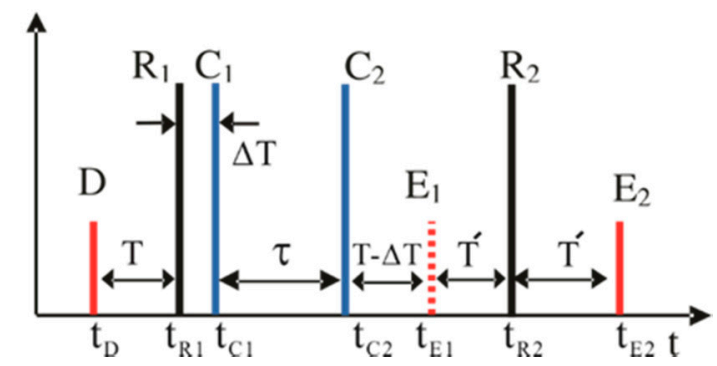

Figure 1. (a) Schematics of controlled double rephasing echoes. (b) Pulse sequence for (a), where $t_{j}$ is the arrival time of pulse $\mathrm{j}$.

The CCC in CDR echoes must be distinguished from resonant Raman or electromagnetically induced transparency (EIT) based on two-photon resonance without shelving on the excited state. For photon echo-based quantum memories, the signal (data) pulse information (phase and amplitude) must be fully transferred into a matter (optical coherence) state via a complete absorption process in an optically dense, inhomogeneously broadened two-level medium [17]. Unlike other coherence optics in the three-level system mentioned above, the inhomogeneity of the ensemble is the fundamental requirement for the coherence evolutions in photon echoes. One unique property of the CCC is the double coherence swapping between the optical and spin states via the control pulse set of $C_{1}$ and $C_{2}$. Unlike EIT, the $R_{1}$ and $C_{1}$ must be differentiated from the two-photon Raman coherence, where the delay $\Delta T$ between $R_{1}$ and $C_{1}$ must be longer than the inverse of inhomogeneous width. Usually, this 
requirement is easily satisfied for the consecutive $\pi$-optical pulse sequence in most rare-earth doped solids [27].

The purpose of $C_{1}$ is simply to hold both optical phase decay via complete population transfer from the excited state $|2\rangle$ to the auxiliary spin state $|3\rangle$, resulting in optical-spin coherence conversion with $\rho_{12}=0$ [11]. For this, the state $|3\rangle$ must be set to be vacant initially. When the second control pulse $C_{2}$ is turned on, the system population is completely recovered to the initial one reached by $R_{1}$. However, the system coherence is not invariant due to the $\pi$-phase shift, resulting in absorptive photon echo $\mathrm{E}_{2}[11-13,18,24-27]$.

The interaction picture Hamiltonian in the atom-field interactions under rotating-wave approximation of the proposed system in Figure 1a is written as

$$
H=-\hbar / 2\left[\begin{array}{ccc}
0 & \Omega_{j} & 0 \\
\Omega_{j} & 0 & \Omega_{k} \\
0 & \Omega_{k} & 0
\end{array}\right]
$$

where $\Omega_{j}\left(j=\mathrm{D}, \mathrm{R}_{1}\right.$, or $\left.\mathrm{R}_{2}\right)$ is the Rabi frequency of $\mathrm{D}, \mathrm{R}_{1}$, and $\mathrm{R}_{2}$, and $\Omega_{k}\left(k=\mathrm{C}_{1}\right.$ or $\left.\mathrm{C}_{2}\right)$ is the Rabi frequency of $C_{1}$ or $C_{2}$. We calculate the rate equations for the density matrix elements using the Von Neumann equation [29].

$$
\dot{\rho}=-\frac{i}{\hbar}[H, \rho]-\frac{1}{2}\{\Gamma, \rho\}
$$

The corresponding rate equations are

$$
\begin{gathered}
\dot{\rho}_{11}=-i \frac{\Omega_{j}}{2}\left(\rho_{12}-\rho_{21}\right), \\
\dot{\rho}_{22}=-i \frac{\Omega_{j}}{2}\left(\rho_{21}-\rho_{12}\right)-i \frac{\Omega_{k}}{2}\left(\rho_{23}-\rho_{32}\right), \\
\dot{\rho}_{33}=-i \frac{\Omega_{k}}{2}\left(\rho_{32}-\rho_{23}\right), \\
\dot{\rho}_{12}=-i \frac{\Omega_{j}}{2}\left(\rho_{11}-\rho_{22}\right)-i \frac{\Omega_{k}}{2} \rho_{13}, \\
\dot{\rho}_{12}=-i \frac{\Omega_{k}}{2} \rho_{12}+i \frac{\Omega_{j}}{2} \rho_{23}, \\
\dot{\rho}_{23}=-i \frac{\Omega_{k}}{2}\left(\rho_{22}-\rho_{33}\right)+i \frac{\Omega_{j}}{2} \rho_{13},
\end{gathered}
$$

where all decay rates are set to zero for simplicity. We now consider the CDR echo scheme for the discussion below. For this, we start with a general DR scheme to investigate the absorptive coherence of the final echo $E_{2}$ without $C_{1}$ and $C_{2}$ pulses in Figure 1.

\section{Discussion}

\subsection{DR Photon Echoes}

In this subsection, we study conventional two-pulse photon echoes in a DR scheme without $C_{1}$ and $\mathrm{C}_{2}$ in Figure 1. We derive time-dependent density matrix equations for the expressions of coherence between the ground and excited states and the population in each bare state.

\subsubsection{D-Pulse}

We first derive the expressions of coherence and population excited by the D-pulse. The equations of motion for D-pulse by setting $\Omega_{j}=\Omega_{\mathrm{D}}$ and $\Omega_{k}=0$ in Equations (3)-(8) are as follows: 


$$
\begin{aligned}
& \dot{\rho}_{11}=-i \frac{\Omega_{\mathrm{D}}}{2}\left(\rho_{12}-\rho_{21}\right), \\
& \dot{\rho}_{22}=-i \frac{\Omega_{\mathrm{D}}}{2}\left(\rho_{21}-\rho_{12}\right), \\
& \dot{\rho}_{12}=-i \frac{\Omega_{\mathrm{D}}}{2}\left(\rho_{11}-\rho_{22}\right), \\
& \dot{\rho}_{21}=-i \frac{\Omega_{\mathrm{D}}}{2}\left(\rho_{22}-\rho_{11}\right) .
\end{aligned}
$$

Initially, all atoms are in the ground state $|1\rangle: \rho_{11}(0)=1 ; \rho_{22}(0)=\rho_{12}(0)=\rho_{21}(0)=0$. The Laplace transform of Equations (9)-(12) with $\rho_{11}+\rho_{22}=1$ yields

$$
\begin{gathered}
\mathcal{L}\left[\rho_{11}\right]=\frac{2 s^{2}+\Omega_{\mathrm{D}}^{2}}{2 s\left(s^{2}+\Omega_{\mathrm{D}}^{2}\right)^{\prime}} \\
\mathcal{L}\left[\rho_{12}\right]=\frac{-i \Omega_{\mathrm{D}}}{\left(s^{2}+\Omega_{\mathrm{D}}^{2}\right)}, \\
\mathcal{L}\left[\rho_{21}\right]=\frac{i \Omega_{\mathrm{D}}}{\left(s^{2}+\Omega_{\mathrm{D}}^{2}\right)} .
\end{gathered}
$$

The final equations for population and coherence are obtained by taking the inverse Laplace transform of Equations (13)-(15).

$$
\begin{gathered}
\rho_{11}=\cos ^{2}\left(\frac{\varphi_{\mathrm{D}}}{2}\right), \\
\rho_{22}=\sin ^{2}\left(\frac{\varphi_{\mathrm{D}}}{2}\right), \\
\rho_{12}=-\frac{i}{2} \sin \left(\varphi_{\mathrm{D}}\right),
\end{gathered}
$$

where $\varphi_{\mathrm{D}}$ is the area of the D-pulse. The D-pulse obeys the area theorem which has a direct relationship with coherence [30].

$$
\frac{\partial \varphi_{\mathrm{D}}}{\partial z}=-\frac{\alpha}{2} \sin \left(\varphi_{\mathrm{D}}\right),
$$

where $\alpha$ is the absorption coefficient. For the D-pulse having a very small area, $\sin \left(\varphi_{\mathrm{D}}\right) \approx 1, \varphi_{\mathrm{D}}=$ $\left(\varphi_{\mathrm{D}}\right)_{0} e^{-\alpha z / 2}$ representing Beer's law. The information of D-pulse is now transferred into the ensemble coherence. For a weak D-pulse $\varphi_{\mathrm{D}} \ll 1$, the atomic population still remains in the ground state $|1\rangle$ : $\rho_{11} \approx 1 ; \rho_{22} \approx 0$. In our analysis, the D-pulse area is set to be $0.1 \pi$.

\subsection{2. $R_{1}$-Pulse}

As soon as the atoms are excited by $\mathrm{D}$, they immediately start to evolve with their own detuningdependent phase velocity until the rephasing pulse $\left(\mathrm{R}_{1}\right.$-pulse) comes. We use Equations (16)-(18) as initial conditions for $\Omega_{j}=\Omega_{\mathrm{R}_{1}}$ and $\Omega_{k}=0$ to solve Equations (3)-(8). The solution of the rate equations for $\mathrm{R}_{1}$-pulse is as follows:

$$
\begin{gathered}
\rho_{11}=\cos ^{2}\left(\frac{\varphi_{\mathrm{D}}+\varphi_{\mathrm{R}_{1}}}{2}\right), \\
\rho_{22}=\sin ^{2}\left(\frac{\varphi_{\mathrm{D}}+\varphi_{\mathrm{R}_{1}}}{2}\right), \\
\rho_{12}=-\frac{i}{2} \sin \left(\varphi_{\mathrm{D}}+\varphi_{\mathrm{R}_{1}}\right),
\end{gathered}
$$


where $\varphi_{R_{1}}$ is the pulse area of $R_{1}$. Equation (22) indicates that the rephasing $\pi$ pulse $R_{1}$ results in a $\pi$ shift in the coherence $\rho_{12}$ initiated by the D-pulse in Equation $(18)$ as shown in Figure 2a: $\left[\rho_{12}\right] \stackrel{\text { R1 }}{\rightarrow}\left[\rho_{12}\right]^{*}$ (see also Appendix $\mathrm{A}$ for $\pi / 2$ pulse area of $\mathrm{D}$ ). All details of detuning-dependent atom phase evolutions and rephasing effects are numerically shown in Figure 4 of [25], where the real parts of $\rho_{12}$ are exactly symmetric, cancelling each other's coherence. The $\pi$ rephasing pulse swaps the population between ground and excited states as shown in Figure 2b, resulting in spontaneous and/or stimulated emission. To overcome the population inversion, a controlled double rephasing concept was developed in the name of CDR echoes [11,12]. For DR echoes, the second $\pi$ optical pulse $R_{2}$ is added to swap the populations again, where the second echo $\mathrm{E}_{2}$ is free from quantum noises. To fully restore the D-pulse transferred coherence, the first echo $E_{1}$ must be erased (or silenced), where the silent echo does not affect the individual coherence evolutions [21-23].

We derive the coherence and population rate equations for $R_{2}$-pulse by replacing $\Omega_{j}$ with $\Omega_{R_{2}}$ and setting $\Omega_{k}=0$ in Equations (3)-(8). We use Equations (20)-(22) as the initial conditions and calculate the expressions for coherence and populations as follows:

$$
\begin{gathered}
\rho_{11}=\cos ^{2}\left(\frac{\varphi_{\mathrm{D}}+\varphi_{\mathrm{R}_{1}}+\varphi_{\mathrm{R}_{2}}}{2}\right), \\
\rho_{22}=\sin ^{2}\left(\frac{\varphi_{\mathrm{D}}+\varphi_{\mathrm{R}_{1}}+\varphi_{\mathrm{R}_{2}}}{2}\right), \\
\rho_{12}=-\frac{i}{2} \sin \left(\varphi_{\mathrm{D}}+\varphi_{\mathrm{R}_{1}}+\varphi_{\mathrm{R}_{2}}\right) .
\end{gathered}
$$

(a)

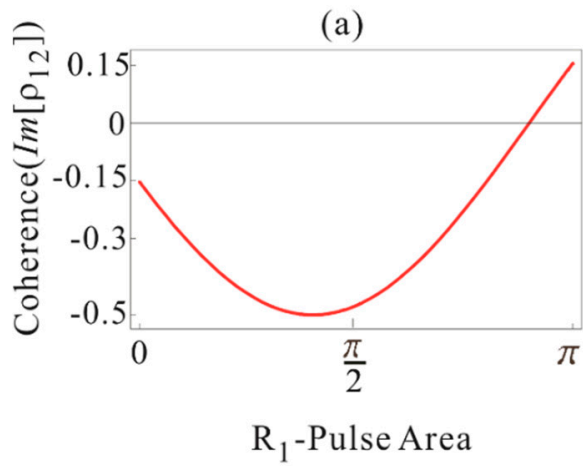

(c)

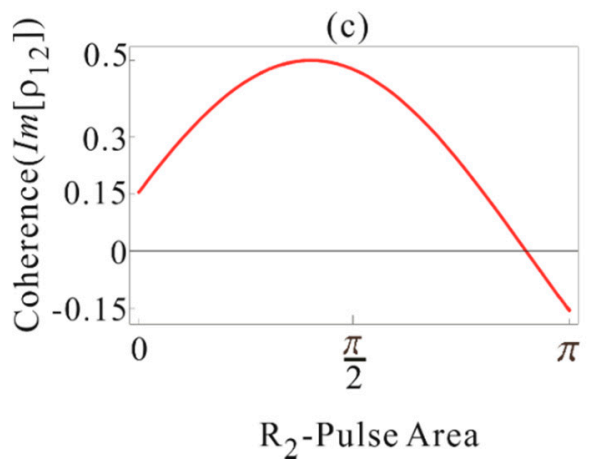

(b)

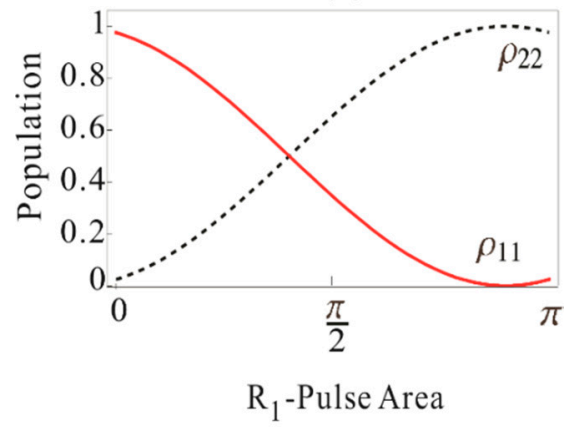

(d)

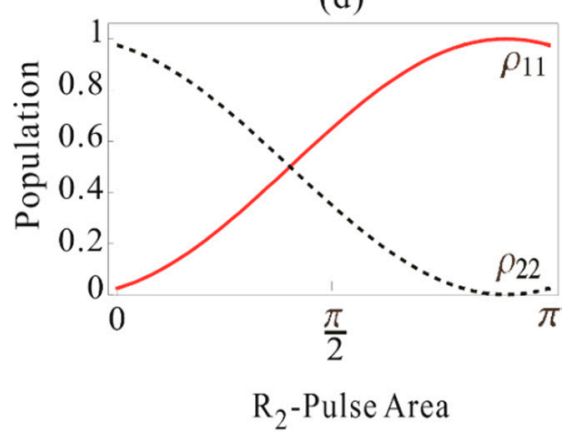

Figure 2. (a) Plot of $\operatorname{Im}\left[\rho_{12}\right]$ (Equation (22)) versus $R_{1}$-pulse area $\varphi_{R_{1}}$ with area of D-pulse $\varphi_{\mathrm{D}}=0.1 \pi$. (b) Corresponding population evolution (red) $\rho_{11}$ (Equation (20)) and (dotted) $\rho_{22}$ (Equation (21)). (c) Plot of $\operatorname{Im}\left[\rho_{12}\right]$ (Equation (25)) versus $\mathrm{R}_{2}$-pulse area $\varphi_{\mathrm{R}_{2}}$ with area of D-pulse $\varphi_{\mathrm{D}}=0.1 \pi$ and that of $\mathrm{R}_{1}$ $\varphi_{R_{1}}=\pi$. (d) Corresponding population evolution (red) $\rho_{11}$ (Equation (23)) and (dotted) $\rho_{22}$ (Equation (24)). 
In Figure $2 c, d$, the $R_{2}$ pulse area-dependent coherence and population are shown for $\varphi_{\mathrm{D}}=0.1 \pi$ and $\varphi_{R_{1}}=\pi$. As shown in Figure $2 c$, the $\pi-R_{2}$ pulse inverts the coherence as the $\pi-R_{1}$ pulse does. Here, the negative sign in the coherence $\rho_{12}$ shows absorption. Thus, the second echo by $\mathrm{R}_{2}$ is absorptive like the data pulse $\mathrm{D}[11,12]$. This means that the generated echo $\mathrm{E}_{2}$ in the DR scheme cannot be radiated out of the medium due to the coherent transient effects, as D is fully absorbed into the medium. By the way, the observations of $E_{2}$ in [21-23] were understood as imperfect rephasing-caused coherence leakage due to Gaussian distributed light pulses [24]. Our aim here is to get the inversion-free emissive echo. To convert the absorptive echo $\mathrm{E}_{2}$ in Figure 2 into an emissive one, the CDR echo scheme is applied. In the section below, we describe the roles of $C_{1}$ and $C_{2}$ for CCC in detail.

\subsection{CDR Photon Echoes}

In this subsection, we discuss the CDR echo of Figure 1 by inserting the control pulse set of $C_{1}$ and $C_{2}$ in the DR scheme. The control pulse set position can be after either $R_{1}$ as shown in Figure $1 b$ or $\mathrm{R}_{2}$ as discussed in [11,12]. In both cases, $\mathrm{C}_{1}$ must be activated before the echo timing [25].

\subsection{1. $\mathrm{C}_{1}$-Pulse}

The function of $\mathrm{C}_{1}$-pulse with a $\pi$-pulse area is to temporally hold optical coherence decay, as well as optical phase evolution, via transferring population in the excited state $|2\rangle$ to an auxiliary ground (spin) state $|3\rangle$. In general, spin phase decay rate is much longer than the optical counterpart in rare-earth doped crystals. Thus, $C_{1}$ plays the role of storage time extension [12,18]. The coherence and population changes by $C_{1}$ can be obtained using Equations (20)-(22) as initial conditions. The solutions of density matrix Equations (3)-(8) for $C_{1}$ are obtained as

$$
\begin{gathered}
\rho_{11}=\cos ^{2}\left(\frac{\varphi_{\mathrm{D}}+\varphi_{\mathrm{R}_{1}}}{2}\right), \\
\rho_{22}=\cos ^{2}\left(\frac{\varphi_{\mathrm{C}_{1}}}{2}\right) \sin ^{2}\left(\frac{\varphi_{\mathrm{D}}+\varphi_{\mathrm{R}_{1}}}{2}\right), \\
\rho_{33}=\sin ^{2}\left(\frac{\varphi_{\mathrm{C}_{1}}}{2}\right) \sin ^{2}\left(\frac{\varphi_{\mathrm{D}}+\varphi_{\mathrm{R}_{1}}}{2}\right), \\
\rho_{12}=-\frac{i}{2} \cos \left(\frac{\varphi_{\mathrm{C}_{1}}}{2}\right) \sin \left(\varphi_{\mathrm{D}}+\varphi_{\mathrm{R}_{1}}\right), \\
\rho_{13}=-\frac{1}{2} \sin \left(\frac{\varphi_{\mathrm{C}_{1}}}{2}\right) \sin \left(\varphi_{\mathrm{D}}+\varphi_{\mathrm{R}_{1}}\right), \\
\rho_{23}=-\frac{i}{2} \sin \left(\varphi_{\mathrm{C}_{1}}\right) \sin ^{2}\left(\frac{\varphi_{\mathrm{D}}+\varphi_{\mathrm{R}_{1}}}{2}\right) .
\end{gathered}
$$

The optical coherence $\rho_{12}$ in Equation (29) by $C_{1}$-pulse is equal to $\cos \left(\varphi_{C_{1}} / 2\right)$ times the coherence generated by $R_{1}$-pulse in Equation (22), where the $R_{1}$-resulted coherence is 0.15 for the $0.1 \pi$ of $D$-pulse and $\pi$ of R1-pulse (see Figure 3). Thus, Equation (29) becomes $\rho_{12}=0.15 \mathrm{i} \cos \left(\varphi_{\mathrm{C}_{1}} / 2\right)$ (see also Figure 2a). Similarly, the spin coherence in Equation (30) is $\rho_{13}=0.15 \sin \left(\varphi_{\mathrm{C}_{1}} / 2\right)$. In the absence of the $\mathrm{C}_{1}$-pulse, i.e., $\varphi_{\mathrm{C}_{1}}=0, \rho_{12}=0.15 i$ and $\rho_{13}=0$. In the presence of the $\pi \mathrm{C}_{1}$-pulse, the optical and spin coherence becomes $\rho_{12}=0.15 i \cos (\pi / 2)=0$ and $\rho_{13}=0.15 \sin (\pi / 2)=0.15 i e^{-i \pi / 2}$, respectively. The $\pi-C_{1}$-pulse adds a $\pi / 2$ phase shift to the transferred coherence $\rho_{13}$ [28]. This is a well-known property in resonant two-field interactions in a three-atomic system, where there is a $\pi / 2$ phase shift between $\operatorname{Im}\left[\rho_{12}\right]$ and $\operatorname{Re}\left[\rho_{13}\right]$ In conclusion, the $\mathrm{C}_{1}$-pulse locks both optical phase decay and coherence evolutions, while it transfers $\rho_{12}$ into $\rho_{13}$ with a $\pi / 2$ phase shift via complete population transfer. In other words, $\operatorname{Im}\left[\rho_{12}\right]$ becomes $\operatorname{Re}\left[\rho_{13}\right]$ as shown in Figure 3a. Here, $\operatorname{Im}\left[\rho_{13}\right]$ is zero as $\operatorname{Re}\left[\rho_{12}\right]$ is zero in Equations (16)-(18). 
(a)

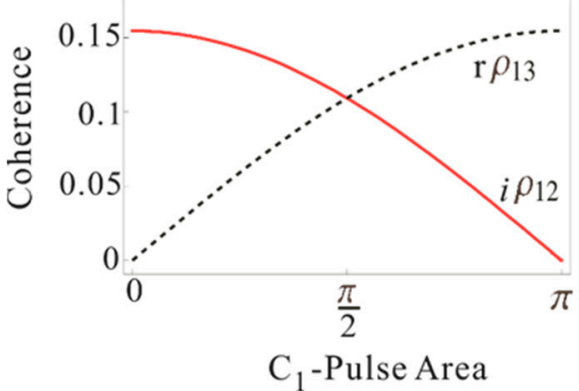

(c)

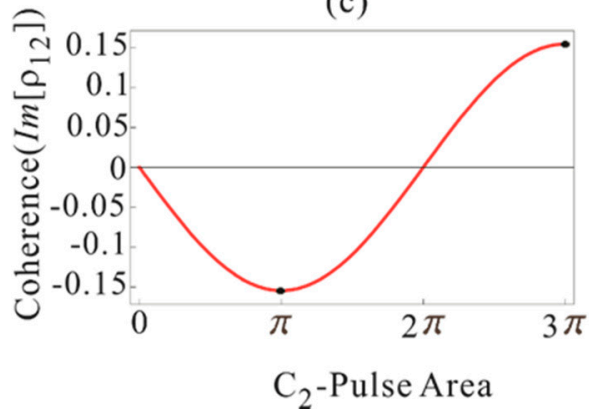

(b)

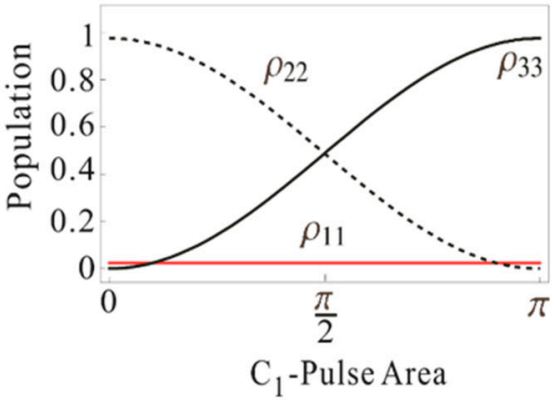

(d)

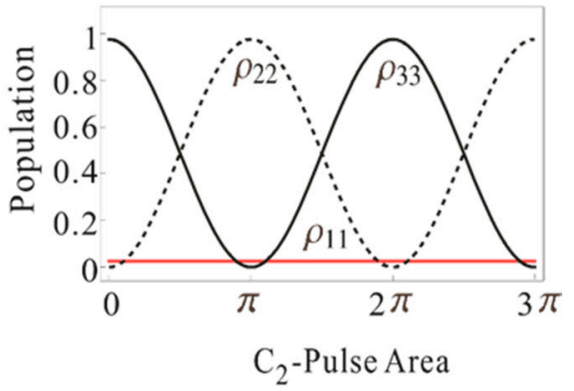

Figure 3. (a) Plot of $\operatorname{Im}\left[\rho_{12}\right]$ and $\operatorname{Re}\left[\rho_{13}\right]$ (Equations (29) and (30)) versus $C_{1}$-pulse area $\varphi_{\mathrm{C}_{1}}$ with area of D-pulse $\varphi_{\mathrm{D}}=0.1 \pi$ and that of $\mathrm{R}_{1} \varphi_{\mathrm{R}_{1}}=\pi$. (b) Corresponding population evolution (red) $\rho_{11}$ (Equation (26)), (dotted) $\rho_{22}$ (Equation (27)), and black $\rho_{33}$ (Equation (28)). (c) Plot of $\operatorname{Im}\left[\rho_{12}\right]$ (Equation (35)) versus $\mathrm{C}_{2}$-pulse area $\varphi_{\mathrm{C}_{2}}$ with area of other pulses $\varphi_{\mathrm{D}}=0.1 \pi, \varphi_{\mathrm{C}_{1}}=\pi$, and $\varphi_{\mathrm{R}_{1}}=\pi$. (d) Corresponding population evolution (red) $\rho_{11}$ (Equation (32)), (dotted) $\rho_{22}$ (Equation (33)), and (black) $\rho_{33}$ (Equation (34)).

\subsection{2. $\mathrm{C}_{2}$-Pulse}

The function of $C_{2}$-pulse is to restore the transferred coherence by $C_{1}$. Using Equations (26)-(31) as the initial conditions, and setting $\Omega_{k}=\Omega_{C_{2}}$ and $\Omega_{j}=0$ in Equations (3)-(8), the system coherence and population expressions by $\mathrm{C}_{2}$-pulse are obtained as follows:

$$
\begin{gathered}
\rho_{11}=\cos ^{2}\left(\frac{\varphi_{\mathrm{D}}+\varphi_{\mathrm{R}_{1}}}{2}\right) \\
\rho_{22}=\cos ^{2}\left(\frac{\varphi_{\mathrm{C}_{1}}+\varphi_{\mathrm{C}_{2}}}{2}\right) \sin ^{2}\left(\frac{\varphi_{\mathrm{D}}+\varphi_{\mathrm{R}_{1}}}{2}\right), \\
\rho_{33}=\sin ^{2}\left(\frac{\varphi_{\mathrm{C}_{1}}+\varphi_{\mathrm{C}_{2}}}{2}\right) \sin ^{2}\left(\frac{\varphi_{\mathrm{D}}+\varphi_{\mathrm{R}_{1}}}{2}\right), \\
\rho_{12}=-\frac{i}{2} \cos \left(\frac{\varphi_{\mathrm{C}_{1}}+\varphi_{\mathrm{C}_{2}}}{2}\right) \sin \left(\varphi_{\mathrm{D}}+\varphi_{\mathrm{R}_{1}}\right), \\
\rho_{13}=-\frac{1}{2} \sin \left(\frac{\varphi_{\mathrm{C}_{1}}+\varphi_{\mathrm{C}_{2}}}{2}\right) \sin \left(\varphi_{\mathrm{D}}+\varphi_{\mathrm{R}_{1}}\right), \\
\rho_{23}=-\frac{i}{2} \sin \left(\varphi_{\mathrm{C}_{1}}+\varphi_{\mathrm{C}_{2}}\right) \sin ^{2}\left(\frac{\varphi_{\mathrm{D}}+\varphi_{\mathrm{R}_{1}}}{2}\right) .
\end{gathered}
$$

The coherence in Equation (35) is equal to $\cos \left(\left(\varphi_{\mathrm{C}_{1}}+\varphi_{\mathrm{C}_{2}}\right) / 2\right)$ multiplied by the coherence excited by $\mathrm{R}_{1}$-pulse in Equation (22). The $\pi-\pi$ pulse sequence of $C_{1}$ and $C_{2}$, therefore, induces a coherence inversion via the round trip of population transfer between the excited and auxiliary states: $\cos ((\pi+\pi) / 2)=-1$ (see Figure 3c) $[11,12,24]: \rho_{12} \stackrel{C_{1} \& C_{2}}{\longrightarrow}-\rho_{12}$. This coherence inversion mechanism is completely different 
from the rephasing by $R_{1}$ or $R_{2}$ [12]. In order to resume the coherence initiated by the $R_{1}$-pulse, the sum pulse area of $C_{1}$ and $C_{2}$ must be equal to $4 n \pi(n=1,2,3 \ldots)$. In Figure $3 c, d$, we plot the coherence and population as a function of the $C_{2}$-pulse area for $\varphi_{D}=0.1 \pi, \varphi_{R_{1}}=\pi$, and $\varphi_{C_{1}}=\pi$. Figure $3 c$ shows that the ensemble coherence excited by $D$ and rephased by $R_{1}$ is recovered with the $3 \pi C_{2}$-pulse. The $3 \pi C_{2}$, of course, returns the population from state $|3\rangle$ to the excited state $|2\rangle$ as shown in Figure $3 \mathrm{~d}$. Thus, the $(\pi-\pi) C_{1}-C_{2}$ pulse sequence in a controlled AFC [31] induces an absorptive echo as in the DR scheme in Figure $2 \mathrm{c}$ due to the $\pi$-phase shift by the control Rabi flopping. The experimental observation in [31] is not an artefact but due to the coherence leakage through imperfect rephasing by commercial Gaussian distributed laser pulses, where its maximum echo efficiency is far less then unity [24]. In the CDR echo scheme, however, the $\pi-\pi$ pulse sequence of $C 1$ and $C 2$ is required to compensate for the $\pi$-phase shift in the DR scheme.

\subsection{3. $R_{2}$-Pulse}

For the CDR echo in Figure 1, the final analytic solutions of density matrix Equations (3)-(8) are obtained using Equations (32)-(37) as the initial conditions.

$$
\begin{aligned}
& \rho_{11}=\frac{1}{16}\left[\cos \left(\frac{\varphi_{\mathrm{C}_{1}}+\varphi_{\mathrm{C}_{2}}-\varphi_{\mathrm{D}}-\varphi_{\mathrm{R}_{2}}-\varphi_{\mathrm{R}_{1}}}{2}\right)-\cos \left(\frac{\varphi_{\mathrm{C}_{1}}+\varphi_{\mathrm{C}_{2}}-\varphi_{\mathrm{D}}+\varphi_{\mathrm{R}_{2}}-\varphi_{\mathrm{R}_{1}}}{2}\right)\right. \\
&+2 \cos \left(\frac{\varphi_{\mathrm{D}}-\varphi_{\mathrm{R}_{2}}+\varphi_{\mathrm{R}_{1}}}{2}\right)-\cos \left(\frac{\varphi_{\mathrm{C}_{1}}+\varphi_{\mathrm{C}_{2}}+\varphi_{\mathrm{D}}-\varphi_{\mathrm{R}_{2}}+\varphi_{\mathrm{R}_{1}}}{2}\right) \\
&\left.+\cos \left(\frac{\varphi_{\mathrm{C}_{1}}+\varphi_{\mathrm{C}_{2}}+\varphi_{\mathrm{D}}+\varphi_{\mathrm{R}_{2}}-\varphi_{\mathrm{R}_{1}}}{2}\right)+2 \cos \left(\frac{\varphi_{\mathrm{D}}+\varphi_{\mathrm{R}_{2}}+\varphi_{\mathrm{R}_{1}}}{2}\right)\right]^{2}, \\
& \rho_{22}=\frac{1}{16}\left[\sin \left(\frac{\varphi_{\mathrm{C}_{1}}+\varphi_{\mathrm{C}_{2}}-\varphi_{\mathrm{D}}-\varphi_{\mathrm{R}_{2}}-\varphi_{\mathrm{R}_{1}}}{2}\right)+\sin \left(\frac{\varphi_{\mathrm{C}_{1}}+\varphi_{\mathrm{C}_{2}}-\varphi_{\mathrm{D}}+\varphi_{\mathrm{R}_{2}}-\varphi_{\mathrm{R}_{1}}}{2}\right)\right. \\
&+ 2 \sin \left(\frac{\varphi_{\mathrm{D}}-\varphi_{\mathrm{R}_{2}}+\varphi_{\mathrm{R}_{1}}}{2}\right)-\sin \left(\frac{\varphi_{\mathrm{C}_{1}}+\varphi_{\mathrm{C}_{2}}+\varphi_{\mathrm{D}}-\varphi_{\mathrm{R}_{2}}+\varphi_{\mathrm{R}_{1}}}{2}\right) \\
&\left.-\sin \left(\frac{\varphi_{\mathrm{C}_{1}}+\varphi_{\mathrm{C}_{2}}+\varphi_{\mathrm{D}}+\varphi_{\mathrm{R}_{2}}-\varphi_{\mathrm{R}_{1}}}{2}\right)-2 \sin \left(\frac{\varphi_{\mathrm{D}}+\varphi_{\mathrm{R}_{2}}+\varphi_{\mathrm{R}_{1}}}{2}\right)\right]^{2}, \\
& \rho_{12}=-\frac{i}{16}[2 \sin \left(\varphi_{\mathrm{R}_{2}}\right) \\
&+2 \sin \left(\varphi_{\mathrm{R}_{2}}\right) \cos \left(\varphi_{\mathrm{D}}+\varphi_{\mathrm{R}_{1}}\right)\left(3+\cos \left(\frac{\varphi_{\mathrm{C}_{1}}+\varphi_{\mathrm{C}_{2}}}{2}\right)\right) \\
&+\sin \left(\varphi_{\mathrm{C}_{1}}+\varphi_{\mathrm{C}_{2}}-\varphi_{\mathrm{R}_{2}}\right) \\
&-\sin \left(\varphi_{\mathrm{C}_{1}}+\varphi_{\mathrm{C}_{2}}+\varphi_{\mathrm{R}_{2}}\right) \\
&\left.+8 \cos \left(\varphi_{\mathrm{R}_{2}}\right) \sin \left(\varphi_{\mathrm{D}}+\varphi_{\mathrm{R}_{1}}+\varphi_{\mathrm{C}_{2}}\right) \cos \left(\frac{\varphi_{\mathrm{C}_{1}}+\varphi_{\mathrm{C}_{2}}}{2}\right)\right] .
\end{aligned}
$$

In Figure 4, we plot the evolutions of coherence and population as a function of $\mathrm{R}_{2}$-pulse area for $\varphi_{\mathrm{D}}=0.1 \pi, \varphi_{\mathrm{R}_{1}}=\pi, \varphi_{\mathrm{C}_{1}}=\pi$, and $\varphi_{\mathrm{C}_{2}}=\pi$. As a result, both coherence and population excited by $\mathrm{D}$ are recovered with a $\pi$-pulse area of $R_{2}$, where spontaneous or stimulated emission-caused quantum noises are completely eliminated.

The present scheme can experimentally be realized in a rare-earth $\mathrm{Pr}^{3+}$-doped $\mathrm{Y}_{2} \mathrm{SiO}_{5}$. In most rare-earth doped media, the ground state hyperfine splitting is a few tens of megahertz. Thus, tens of $\mathrm{GHz}$ in an optical inhomogeneous width can be sliced for multiple spectral channels for multimode quantum memory applications, where the practical parameter of optical Rabi frequency is $\sim \mathrm{MHz}$. For an extended storage time by $\mathrm{C}_{1}$, Zeeman states may be used [32].

In an atomic ensemble such as $\mathrm{Rb}$ vapors, Zeeman splitting may also be used, where optical polarization control is adapted to form a three-level system. However, such an atomic medium may not a good candidate for the photon-echo-based quantum memory applications simply due to fast atomic diffusion. Moreover, providing a $\pi$ optical pulse in a few-ns pulse duration within the optical phase decay time is very challenging with a commercial continuous wave (CW) laser system. 
(a)

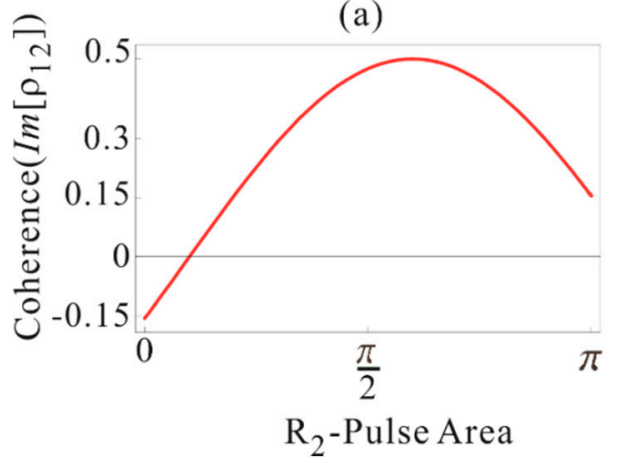

(b)

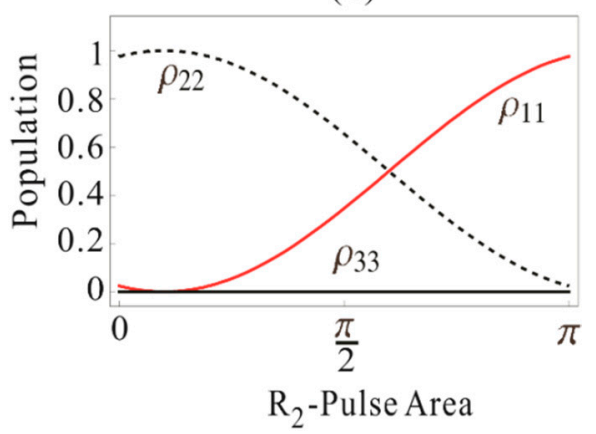

Figure 4. (a) Plot of $\operatorname{Im}\left[\rho_{12}\right]$ (Equation (41)) versus $R_{2}$-pulse $\varphi_{R_{2}}$. The area of other pulses are $\varphi_{D}=0.1 \pi$, $\varphi_{R_{1}}=\pi, \varphi_{C_{1}}=\pi$, and $\varphi_{C_{2}}=\pi$. (b) Corresponding population evolution (red) $\rho_{11}$ (Equation (38)), (dotted) $\rho_{22}$ (Equation (39)), and (black) $\rho_{33}$ (Equation (40)).

\section{Conclusions}

In conclusion, we analytically presented the CDR echo protocol for spontaneous emission-freephoton echo-based quantum memory applications by combining double rephasing photon echoes with control Rabi flopping. For this, time-dependent density matrix equations were analytically solved for coherence/population evolutions to investigate the phase shift of a resonant atom. To overcome the absorptive echo problem in a bare double rephasing photon echo scheme, a consecutive $\pi-\pi$ control pulse sequence is inserted right after the first rephasing pulse. The control pulse-generated $\pi$-phase shift was exactly compensated for with another $\pi$-phase shift resulting from the double rephasing scheme. As a result, emissive photon echoes were obtained under no population inversion.

Author Contributions: The original draft was written by R.U., and reviewed and edited by B.S.H. All listed authors meet the ICMJE criteria. We attest that all authors contributed significantly to the creation of this manuscript, each having fulfilled criteria as established by the ICMJE. We confirm that the order of authors listed in the manuscript was approved by all named authors. All authors have read and agreed to the published version of the manuscript.

Funding: ICT R\&D program of MSIP/IITP (1711028311: Reliable crypto-system standards and core technology development for secure quantum key distribution network) and the GRI research grant of GIST in 2020.

Conflicts of Interest: The authors declare no conflict of interest.

\section{Appendix A}

In the Appendix $A$, we analyze the coherence swapping by the $\pi$ control pulse $C_{1}$ for the $\pi / 2$ D-pulse in Figure 3. The $\pi / 2$ D-pulse creates maximum coherence in the $|1\rangle \leftrightarrow|2\rangle$ transition, where $\operatorname{Im}\left[\rho_{12}\right]=-0.5$ (see Equation (18) for $\mathrm{D}=\pi / 2$ ). The negative sign represents absorption of the D-pulse. In Figure A1, we show a phase shift of the D-pulse excited coherence according to each applied pulses. The first rephasing pulse $\mathrm{R}_{1}$ switches the coherence from absorption to emission as shown in Figure A1a. Identical $C_{1}$ and $C_{2}$ pulses with $\pi$-pulse area each invert the sign of coherence obtained by $R_{1}$, resulting in an absorptive echo in Figure $A 1 b, c$. Finally, the second rephasing pulse $R_{2}$ adds a $\pi$-phase shift to make the emissive photon echo under no population inversion, as shown in Figure A1d. Either a control pulse set or a double rephasing pulse set with a $2 \pi$ pulse area each does not change the D-excited population distribution. Thus, the CDR echo is confirmed for a noise-free quantum memory protocol. 
(a)

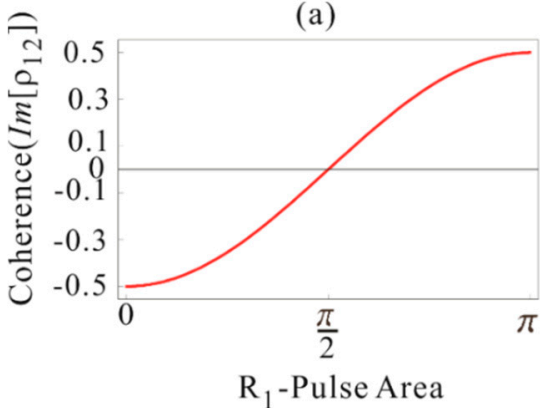

(c)

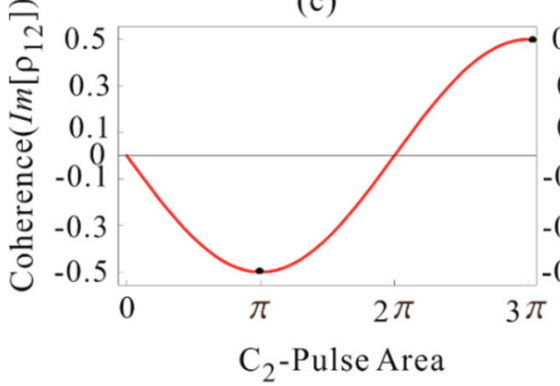

(b)

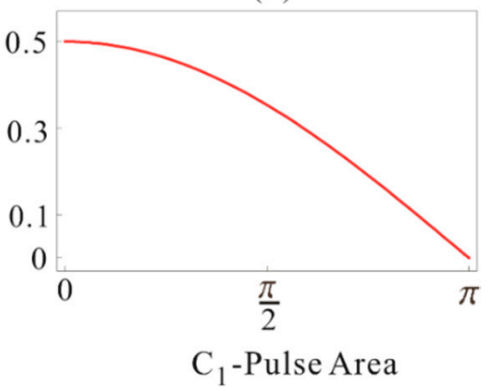

(d)

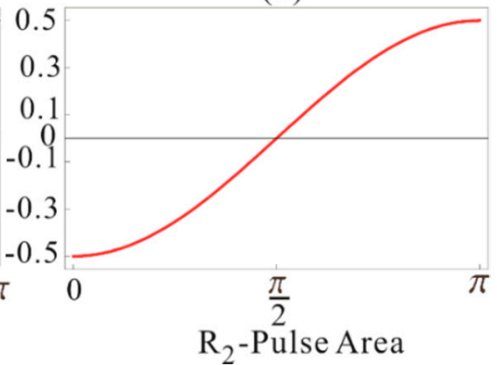

Figure A1. (a) Plot of $\operatorname{Im}\left[\rho_{12}\right]$ versus (a) $\mathrm{R}_{1}$-pulse area $\varphi_{R_{1}}$ (Equation (22)), (b) $C_{1}$-pulse area $\varphi_{C_{1}}$ (Equation (29)), (c) $C_{2}$-pulse area $\varphi_{C_{2}}$ (Equation (35)), and (d) $R_{2}$-pulse area $\varphi_{R_{2}}$ (Equation (41)). The area of the D-pulse is $\varphi_{D}=\pi / 2$.

\section{References}

1. Moiseev, S.A.; Kröll, S. Complete reconstruction of the quantum state of a single-photon wave packet absorbed by a Doppler-broadened transition. Phys. Rev. Lett. 2001, 87, 173601. [CrossRef]

2. Moiseev, S.A.; Tarasov, V.F.; Ham, B.S. Quantum memory photon echo-like techniques in solids. J. Opt. B Quantum Semiclassical Opt. 2003, 5, S497-S502. [CrossRef]

3. Kurnit, N.A.; Abella, I.D.; Hartmann, S.R. Observation of a photon echo. Phys. Rev. Lett. 1964, $13,567$. [CrossRef]

4. Wootter, W.K.; Zurek, W.H. A single quantum cannot be cloned. Nature 1982, 299, 802. [CrossRef]

5. Togan, E.; Chu, Y.; Trifonov, A.; Jiang, L.; Maze, J.; Childress, L.; Dutt, M.V.G.; Sørensen, A.S.; Hemmer, P.R.; Zibrov, A.; et al. Quantum entanglement between an optical photon and a solid-state spin qubit. Nature 2010, 466, 730. [CrossRef]

6. Macfarlane, M.R.; Shelby, R.M. Coherent transient and holeburning spectroscopy of rare earth ions in solids. In Spectroscopy of Solids Containing Rare Earth Ions; Kaplyanskii, A., Macfarlane, R.M., Eds.; Elsevier: Amsterdam, The Netherlands, 1987.

7. Riedmatten, H.; Afzelius, M.; Staudt, M.U.; Simon, C.; Gisin, N. A solid-state light-matter interface at the single-photon level. Nature 2008, 456, 773. [CrossRef]

8. Tittel, W.; Afzelius, M.; Chanelière, T.; Cone, R.; Kröll, S.; Moiseev, S.; Sellars, M.J. Photon-echo quantum memory in solid state systems. Laser Photon. Rev. 2010, 4, 244. [CrossRef]

9. Hétet, G.; Longdell, J.J.; Alexander, A.L.; Lam, P.K.; Sellars, M.J. Electro-optic quantum memory for light using two-level atoms. Phys. Rev. Lett. 2008, 100, 023601. [CrossRef]

10. Hedges, M.P.; Longdell, J.J.; Li, Y.; Sellars, M.J. Efficient quantum memory for light. Nature 2010, 465, 1052. [CrossRef]

11. Ham, B.S. Atom phase controlled noise-free photon echoes. arXiv 2011, arXiv:1101.5480v2.

12. Ham, B.S. Collective atom phase controls in photon echoes for quantum memory applications I: Population inversion removal. Adv. Appl. Sci. Res. 2018, 9, 32.

13. Ham, B.S. A wavelength-convertible quantum memory: Controlled echo. Sci. Rep. 2018, 8, 10675. [CrossRef]

14. Duan, L.-M.; Lukin, M.D.; Cirac, J.I.; Zoller, P. Long-distance quantum communication with atomic ensembles and liner optics. Nature 2001, 414, 413. [CrossRef] 
15. Sangouard, N.; Simon, C.; Riedmatten de, H.; Gisin, N. Quantum repeaters based on atomic ensembles and linear optics. Rev. Mod. Phys. 2011, 83, 33. [CrossRef]

16. Longdell, J.J.; Fraval, E.; Sellars, M.J.; Manson, N.B. Stopped light with storage times greater than one second using electromagnetically induced transparency in a solid. Phys. Rev. Lett. 2015, 95, 063601. [CrossRef]

17. Ham, B.S. Ultralong quantum optical data storage using an optical locking technique. Nat. Photon. $2009,3,518$. [CrossRef]

18. Ham, B.S. Coherent control of collective atom phase for ultralong, inversion-free photon echoes. Phys. Rev. A 2012, 85, 049905. [CrossRef]

19. Langer, L.; Poltavtsev, S.V.; Yugova, I.A.; Salewski, M.; Yakovlev, D.R.; Karczewski, G.; Wojtowicz, T.; Akimov, I.A.; Bayer, M. Access to long-term optical memories using photon echoes retrieved from semiconductor spin. Nat. Photon. 2014, 8, 851. [CrossRef]

20. Ham, B.S. Atom phase-locked coherence conversion using optical locking for ultralong photon storage beyond the spin T2 constraint. New J. Phys. 2012, 14, 013003. [CrossRef]

21. Damon, V.; Bonarota, M.; Louchet-Chauvet, A.; Chanelière, T.; Le Gouët, J.-L. Revival of silenced echo and quantum memory for light. New J. Phys. 2011, 13, 093031. [CrossRef]

22. Arcangeli, A.; Ferrier, A.; Goldner, P. Stark echo modulation for quantum memories. Phys. Rev. A 2016, 93, 062303. [CrossRef]

23. McAuslan, D.L.; Ledingham, P.M.; Naylor, W.R.; Beavan, S.E.; Hedges, M.P.; Sellars, M.J.; Longdell, J.J. Photon-echo quantum memories in inhomogeneously broadened two-level atoms. Phys. Rev. A 2011, 84, 022309. [CrossRef]

24. Ham, B.S. Gaussian beam profile effectiveness on double rephasing photon echoes. arXiv 2017, arXiv:1701.04291.

25. Ham, B.S. Control of photon storage time using phase locking. Opt. Exp. 2010, 18, 1704. [CrossRef]

26. Ullah, R.; Ham, B.S. Understanding of collective atom phase control in modified photon echoes for a near-perfect storage time-extended quantum memory. Entropy 2020, 22, 900. [CrossRef]

27. Hahn, J.; Ham, B.S. Rephasing halted photon echoes using controlled optical deshelving. New J. Phys. 2011, 13, 093011. [CrossRef]

28. Zhong, M.; Hedges, M.; Ahlefeldt, R.; Bartholomew, J.G.; Beavan, S.E.; Wittig, S.M.; Longdell, J.L.; Sellars, M.J. Optical addressable nuclear spin in a solid with a six-hour coherence time. Nature 2015, 517, 177. [CrossRef]

29. Sargent, M., III; Scully, M.O.; Lamb, W.E., Jr. Laser Physics; Addison-Wesley: Boston, MA, USA, 1974.

30. Hahn, E.L.; Shiren, N.S. Application of the area theorem to phonon echoes. Phys. Lett. A 1971, $37,265$. [CrossRef]

31. Afzelius, M.; Usmani, I.; Amari, A.; Lauritzen, B.; Walther, A.; Simon, C.; Sangouard, N.; Minář, J.; De Riedmatten,H.; Gisin, N.; et al. Demonstration of atomic frequency comb memory for light with spin-wave storage. Phys. Rev. Lett. 2010, 104, 040503. [CrossRef]

32. Fraval, E.; Sellars, M.J.; Longdell, J.J. Method of extending hyperfine coherence times in Pr3+:Y2SiO5. Phys. Rev. Lett. 2004, 922, 077601. [CrossRef]

(C) 2020 by the authors. Licensee MDPI, Basel, Switzerland. This article is an open access article distributed under the terms and conditions of the Creative Commons Attribution (CC BY) license (http://creativecommons.org/licenses/by/4.0/). 